J. Perinat. Med. 14 (1986) 421

\title{
An adaptive on-line method for the extraction of the complete fetal electrocardiogram from cutaneous multilead recordings
}

\author{
Dirk Callaerts ${ }^{1}$, Jan Vanderschoot ${ }^{1}$, Joos Vandewalle ${ }^{1}$, Willy Sansen ${ }^{1}$, Gaston \\ Vantrappen ${ }^{2}$, and Jozef Janssens ${ }^{2}$ \\ ${ }^{1}$ ESAT Laboratory, Department of Electronical Engineering, and \\ ${ }^{2}$ Laboratory of G. I. Mobility, Department of Medical Research, University \\ of Leuven, Belgium
}

\section{Introduction}

The task of obtaining a fetal ECG (FECG) from cutaneous recordings is in a so-called inverse problem. This problem consists in characterizing the (internal) bioelectric sources that have generated a given set of (external) measurements. Progress for the FECG has not matched that for the adult ECG. This may be attributed to the following facts:

1) cutaneous recordings of the FECG are disturbed by relatively large noise signals from electrodes, striated muscles etc.;

2) the omnipresent maternal ECG (MECG);

3) the large variability of the way cutaneous potentials are generated by the internal bioelectric sources, due to differences between subjects, changes depending on the stage of pregnancy, movement of the fetus, etc.

Assume that 3 signals from cutaneous electrodes are sampled. At each sampling instant, 3 potential differences are obtained. Such a "sample", consisting of 3 values, can be represented by a single point in a three dimensional frame of reference. For that purpose an origin must be chosen, and to each measurement signal a reference axis must be associated. It is legitimate to call such a "sample" a measurement vector sample. Equivalently an arrow,

\section{Curriculum vitae}

Dirk Callaerts was born in Leuven, Belgium in 1961. He received the Engineering Degree in Applied Sciences from the Katholieke Universiteit Leuven Belgium in 1984. Currently, he is preparing his doctoral thesis in signal processing. The research project is sponsored by the Belgian I. W.O.N.L.

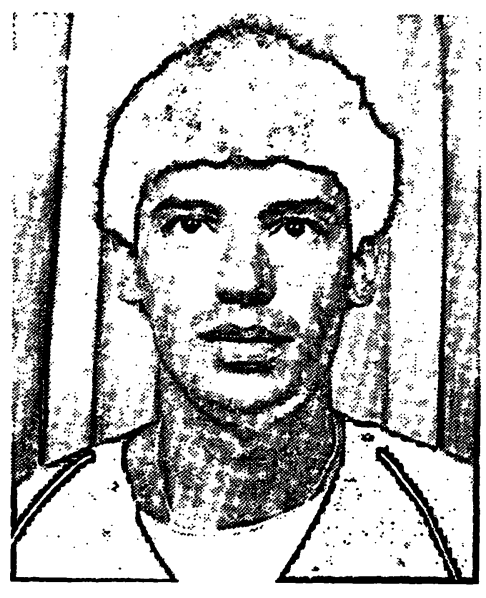

pointing from the origin to the point, could be used in a drawing. The important point is that the components of the vector along the reference axes are the respective measurement values. In the chosen frame of reference, a set of such vector samples can be represented by a cloud of points. Such a set could result from taking measurement vector samples at different time instants.

A good approximation of the way these measurement vector samples are generated can be cast into the following formulation. The electromagnetic basis for this approximation is the linearity, and the quasistatic behaviour of the medium, which is explained in e.g. [10]. 
More details of the geometric representation given below can be found in [13]. To each bioelectric current source within the body, a fixed vector in the frame of reference (the transfer vector) is associated. This vector is completely determined by the geometry of the body, electrode and source positions, and conductivities of the body tissues. A measurement vector sample is obtained by summing vector contributions, one for each source. The vector contribution of a certain source is a vector which has the same or the opposite direction of its associated transfer vector. The length of such a contribution is proportional to the length of the transfer vector and to the instantaneous current of the source. The direction is determined by the sign of the current, being positive or negative. A representation of this is given in figure 1.

To avoid any confusion it should be mentioned that the number of sources that can be "observed" with cutaneous electrodes, regardless of their number, is not equal to the number of internal microscopic bioelectric sources. The main reasons for these limitations are
1) the fact that electrode positions are allowed only on the surface enclosing the volume containing the sources,

2) measurement noise, and

3) cooperation (or correlations) between different microscopic sources.

For example, in [4] it is shown that for realistic noise levels, and using 102 electrode signals, only about 9 adult ECG sources can be observed. In other words in such a 102 dimensional measurement vector space, only 9 transfer vectors can be associated with the heart source. Reducing the number of electrodes may result in a reduction of the number of observed ECG sources, but this is not necessarily so. E. g. the vector cardiographic lead systems have been designed with the aim of observing only 3 "equivalent" ECG sources. Concerning the FECG, to our knowledge, nothing has ever been published about the number of observable sources. The inverse problem can now be reformulated as: given one or more measurement vector samples, determine the length and sign

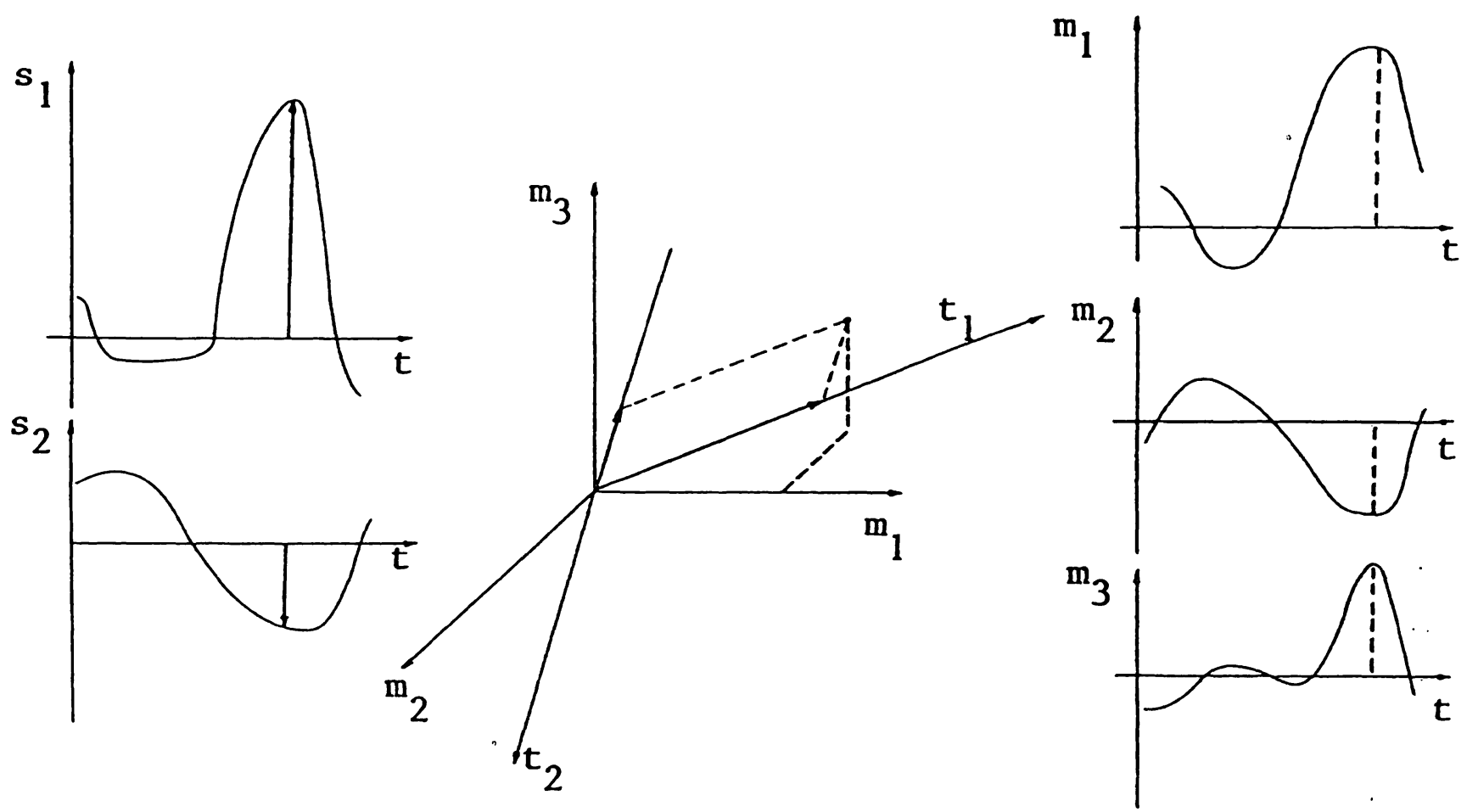

Figure 1. A measurement vector sample is generated by summing the vector contributions of two current sources $s_{1}$ and $s_{2}$ in the direction of the transfer vectors $t_{1}$ and $t_{2}$, each corresponding to a current source. This measurement vector sample is projected on the axes (corresponding to the measurements $m_{1}, m_{2}$ and $m_{3}$ ) of a frame of reference and its components are the actual measured values. 
of vector contributions with the direction of the transfer vectors.

This mathematical problem is similar to solving a set of simultaneous equations or inverting a matrix. It has a unique solution if the transfer vectors satisfy certain conditions. E. g. a sufficient though not necessary condition is that each transfer vector be perpendicular to all others. Practically speaking the solvability of the inverse problem can be improved by choosing appropriate electrode locations. It can be proved that a large number of electrodes is not necessarily better than a small number, as far as solvability is concerned. This fact has not always been appreciated in the literature.

Anyway, if a solution exists, then a signal proportional to a certain source signal can be obtained by projecting each measurement vector sample orthogonally onto the direction of a fixed weight vector. For each transfer vector

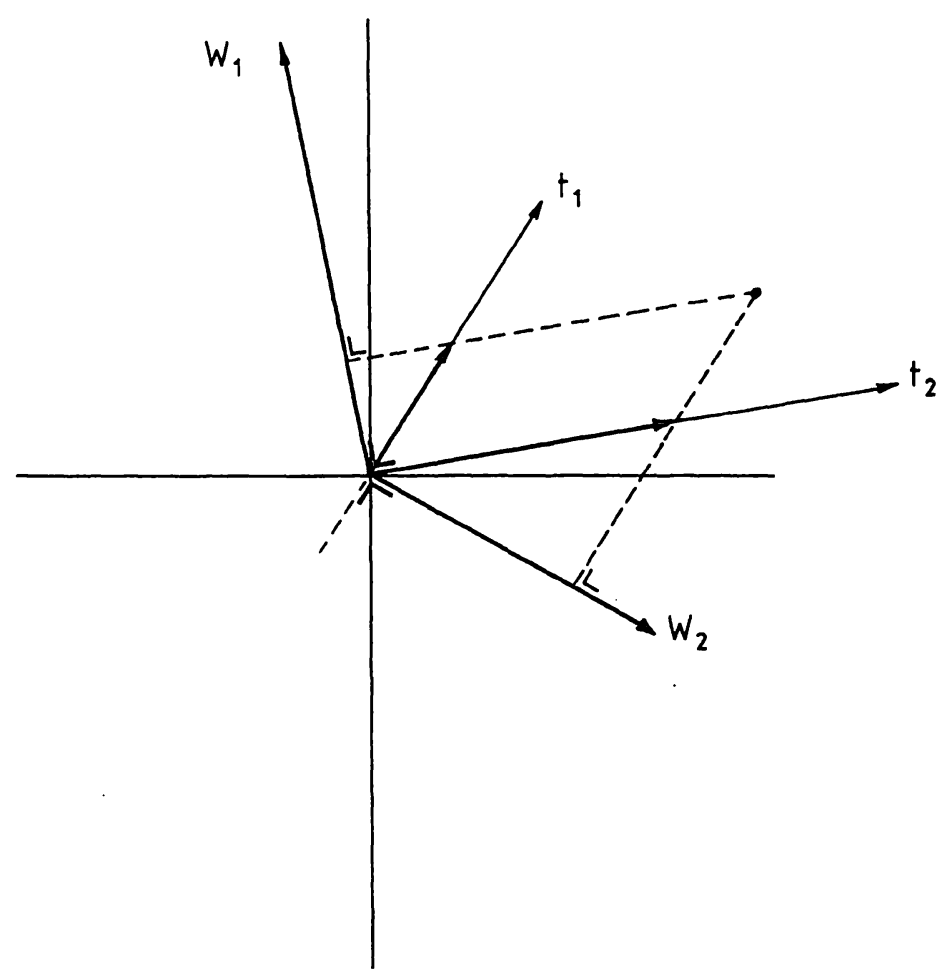

Figure 2. With each transfer vector $t_{i}$ a weight vector $w_{i}$ is assocated that is perpendicular to all other transfer vectors. It is clear that any vector contribution in the direction of $t_{2}\left(t_{1}\right)$ does not change anything about the projection onto $w_{1}\left(w_{2}\right)$. there exists such a weight vector. This is illustrated in figure 2. The computation of such a projection is quite simple, it is a weighted sum of the components of a measurement vector sample. The weight of the $\mathrm{i}$-th measurement component is equal to the $\mathrm{i}$-th component of the weight vector.

Methods aiming at recording the FECG from cutaneous electrodes can be divided into two classes. Some methods resort to procedures in which measurement samples are combined with samples from the past, to obtain an improved FECG signal. These methods use coherent averaging, matched filters, auto- and crosscorrelation techniques, adaptive filters, sequenced adaptive filters, etc. Some main references for this approach are [1], [5], [14]. Other methods use the a priori knowledge that it may be possible to obtain a "clear" FECG signal by means of a weighted sum of signals from distinct electrodes, as explained above. These methods also result in a data reduction: whatever the number of measurement signals, the number of resulting signals is equal to the number of observed sources. In fact this knowledge has resulted in the well known adult VCG lead systems. But, whereas for the adult ECG inverse problem transfer vectors are reasonably predictable, this is not the case for the FECG inverse problem. Some main references for this second class of methods are [2], [7], [8], [11], [12], [13].

As far as we are aware, published methods fail more or less with respect to the following considerations.

1. There is no experimental evidence that the cutaneously observed fetal electrical activity can be represented by only one signal. Therefore, equivalently to the adult ECG, attention must be paid to obtain a complete FECG. This may require more than one signal.

2. For application in a clinical environment, the method may not be too sensitive to actual electrode location, human interpretation of recorded signals, stage of pregnancy, position of fetus, etc. 


\section{Basic principles of the method}

In this section the fundamentals of the proposed method are explained in geometric terms. To start with, a very simplified model is used to introduce the main concepts. Suppose that the maternal electrical heart activity as well as the fetal electrical heart activity could be described completely by one signal each. This would be the case if both hearts acted as one dipole with fixed direction. Further more, assume that on this simplified model, three potential differences are recorded and sampled. Then, as explained above, the set of measurement vector samples can be represented as a cloud of points in a three dimensional frame of reference. For this simplified model, both hearts are observed as a single source. Therefore there is one maternal and one fetal transfer fector. The strong maternal source will push measurement vector samples far away from the origin, in the direction of the maternal transfer vector. The much weaker fetal source causes the measurement vector samples to deviate slightly from the direction of the maternal vector, in the direction of the fetal transfer vector. It is as if the cloud is stretched in the direction of the maternal transfer vector. One way to characterize such a stretching (a mathematically attractive way) is the so called energy in a direction or oriented energy.

The energy in a certain direction, of a set of vector samples, is obtained by projecting orthogonally all the samples onto that direction, and summing the squares of the lengths of these projections. One can imagine the search for the direction with absolutely maximal oriented energy, in order to find the maternal transfer vector. From now on, the unit length vector in the direction of maximal oriented energy will be called the first singular basis vector. The square root of the maximal oriented energy will be called the first singular value.

Suppose that the maternal transfer vector coincides exactly with the first singular basis vector. (In practice these two vectors will be always very close, see [13]). It is crucial to observe that under this condition, projections of the measurement vector samples onto any direction orthogonal to the first singular basis vector contain no contribution whatsoever of the maternal source. Moreover, if the fetal transfer vector does not coincide with the maternal transfer vector, such projections contain contributions from the fetal source only. In other words, a weighted sum of the three measurement signals, with an associated weight vector orthogonal to the first singular basis vector, will result in a signal which is completely free of MECG, and will eventually contain FECG.

All possible directions orthogonal to the first singular basis vector are in a plane. Within this plane, the direction of maximal energy can again be searched for. From now on, equivalently to the above we will use the terms second singular basis vector and second singular value.

By definition, the second singular basis vector is orthogonal to the first, and the second singular value is smaller than the first. For our application it is important to remark that projections onto the direction of the second singular basis vector contain a maximal fetal contribution under the constraint to be free of a maternal contribution. Some of the concepts here introduced are illustrated in figure 3. Projections onto a direction orthogonal to the first as well as the second singular basis vector will be identically zero. This stems from the fact that all measurement vector samples fall in the plane defined by the maternal and the fetal transfer vector.

Consider now the more realistic assumption that each measurement signal also contains noise. Suppose that all these noise signals have a zero mean value, and the same standard deviation. Furthermore these noise signals are completely unpredictable ("white"), and not correlated to each other. It is then clear that in all directions the contribution of these noise signals to the oriented energy is the same, provided that enough vector samples are taken. Practically speaking, enough means that the number of vector samples should exceed say 10 times the number of measurement signals. If 


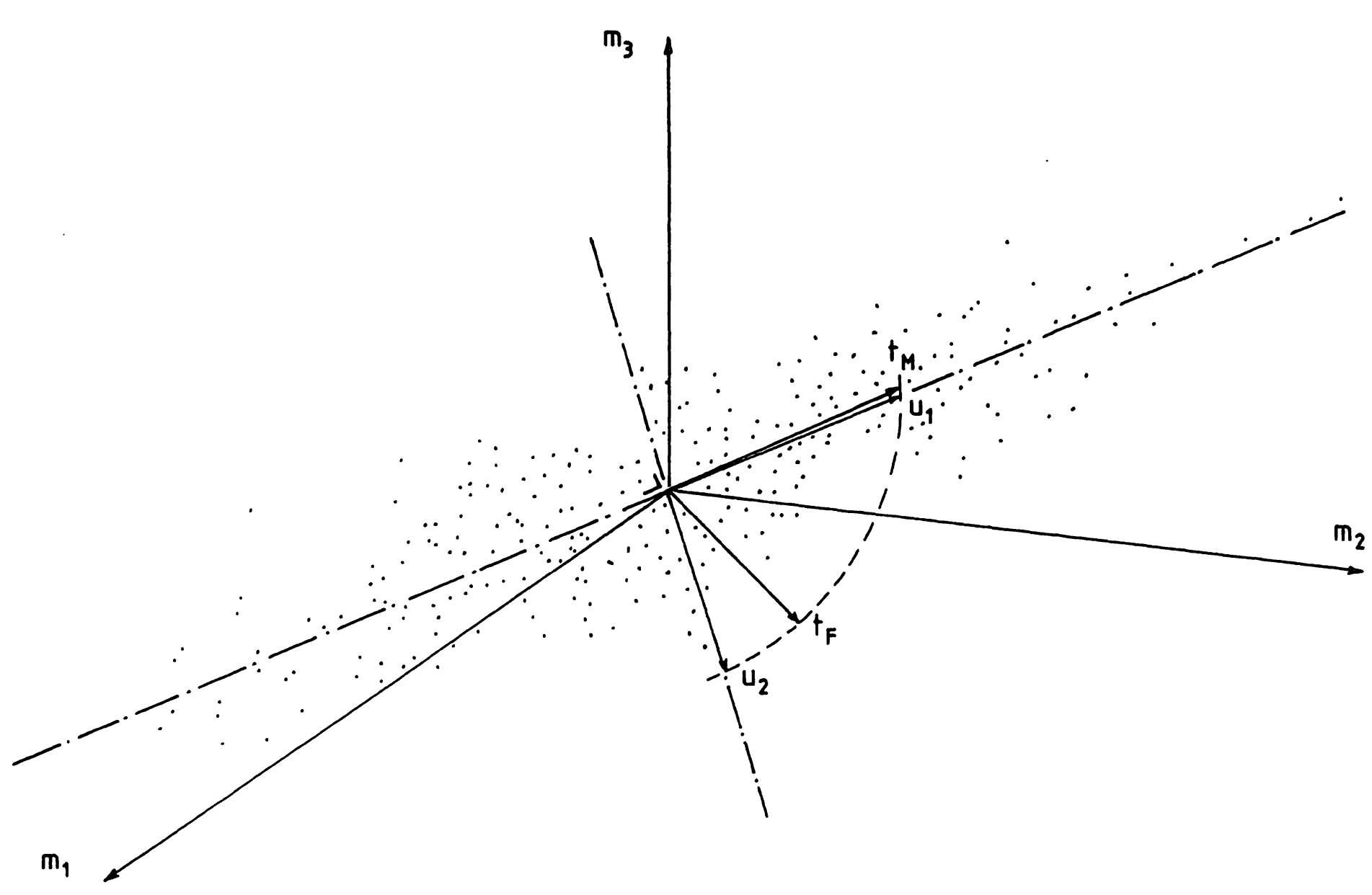

Figure 3. A cloud of $\mathbf{3 0 0}$ samples is represented in a 3-dimensional frame of reference. The transfer vectors $t_{M}$ and $t_{F}$ corresponding to the maternal and the fetal heart, as well as the singular basis vectors $u_{1}$ and $u_{2}$ are shown, for this simulation example. Notice how close $u_{1}$ and $t_{1}$ are.

such a provision is made, the consequence is that the directions of the singular basis vectors will not be influenced by the noise.

As mentioned before, the transfer vectors are influenced by the electrode locations. From the discussion above it should be clear that the projections onto the second singular basis vector contain a maximal FECG contribution in the case that the fetal transfer vector is orthogonal to the maternal transfer vector. Therefore in that case the signal to noise ratio in the resulting signal will also be maximal. One can use this observation in the measurement set up by striving for orthogonality during the search for appropriate locations for the electrodes.

Until now the concern was with a very simplified model. In practice, the number of observed maternal ECG sources is not one, but at least two (an equivalent dipole remaining in a plane). Also the number of observed FECG sources should not be restricted to one. Instead of a single transfer vector, a subspace can be associated with each heart source. Suppose now that for each direction within the maternal subspace, the contributions to the oriented energy of the maternal heart are much larger than those of the fetal heart. The procedure for the separation is then very similar to that for the simplified model. The first singular basis vector can be determined, and it will be in the maternal subspace. A singular basis vector is now defined as follows. The $\mathrm{i}$-th singular basis vector is the unit length vector, constrained to be orthogonal to the first until the (i-1)-th singular basis vector, and for which the oriented energy is maximal. 
Suppose that the dimension of the maternal subspace is $d_{M}$. Then the projections onto the singular basis vectors from the $\left(d_{M}+1\right)$ th on will be completely free of MECG.

If electrodes can be positioned such that every possible vector in the fetal subspace is orthogonal to every possible vector in the maternal subspace, projections onto the $\left(d_{M}+1\right)$ th singular basis vector contain a maximal possible FECG contribution. Moreover, if the fetal subspace has dimension $\mathrm{d}_{\mathrm{F}}$, then projections onto singular basis vectors $d_{M}+1$ until $\mathrm{d}_{\mathrm{M}}+\mathrm{d}_{\mathrm{F}}$ will all contain a FECG contribution. Here again, in any direction the noise will have the same energy contribution, provided enough vector samples are used to determine singular basis vectors.

As a conclusion, the number of electrodes, and their location should be chosen such that the maternal subspace and the fetal subspace are orthogonal to each other. In that case the procedure of determining singular basis vectors and using these vectors as weight vectors, will result in a number of pure MECG signals, a number of pure FECG signals, and a number of noise signals. If the orthogonality is not completely satisfied, some resulting signals will contain a mixture of FECG and MECG signals.

\section{Some practical considerations}

For the actual calculations of singular basis vectors many alternatives are possible. Indeed this is an optimization problem for which many software libraries offer a solution. However, the method considered to be superior to all others in many respects is the so-called singular value decomposition (SVD) of a matrix (for a detailed description, see [6], [9]). The matrix to be decomposed consists of the samples from the measurement signals. It has one row for each measurement signal, and one column for each sampling instant (for more details, see [11], [12], [13]). The procedure presented there is to first collect such a matrix, then execute a singular value decomposition with the GoLUB-REINSCH method [6], and finally to determine the weight- ed sums of measurement signals with the singular basis vectors as weight vectors. These weight vectors are kept fixed. The problems with this procedure are:

1. The calculation can only start when a representative number of measurement vector samples is available.

2. The GoluB-REINSCH method, though ideally suited for the general SVD problem, requires too much calculation time. This is mainly due to the fact that a priori knowledge can not be incorporated.

3. The weight vectors are kept constant. It would be recommended to adapt the weight vectors whenever the transfer vectors change in the course of a recording session.

In the next section a method is presented which inherits the properties of the method in [11], [12], [13], except that it overcomes the three above mentioned problems. Given a good method to calculate the singular value decomposition, the main concern becomes to select measurement vector samples in a good way. Therefore the selection of electrode locations, and the selection of sampling instants is discussed in the following paragraphs.

The benefit of the use of thoracic leads has been questioned many times. Two main reasons have been put forward to advocate the use of abdominal electrode signals only. Firstly, since the number of electrodes is limited due to practical reasons, it seems to be obvious to put these close to the source of interest. A better signal to noise ratio in the resulting signal may then be expected. Secondly, thoracic electrode signals may introduce maternal ECG contributions which would otherwise not have been observed. For example, equivalent quadrupole components of the maternal heart may be introduced. Concerning the first argument, many electrodes close to the fetus serve no useful purpose if fetal transfer vectors are close to maternal transfer vectors. If only abdominal electrodes are used it is expected that hardly anything can be predicted about the fetal transfer vectors, and consequently about their angles with maternal transfer vectors. This is due to 
the large variability of fetal heart position and orientation, and of the geometry of the abdomen during pregnancy. On the contrary if also thoracic signals are used, it can be expected that

1. maternal transfer vectors are almost confined to the subspace of the reference axes of the thoracic measurement signals,

2. fetal transfer vectors are almost confined to the subspace of the reference axes of the abdominal measurement signals.

This is due to the fact that, whatever the strength of a source, its influence on a measurement signal decreases dramatically as the distance to the electrodes increases. These expectations have been confirmed experimentally as reported in [12], [13]. An additional advantage is that the problems of 1) optimal locations for MECG elimination, and 2) optimal locations for FECG detection are decoupled now. Concerning the second argument, about introducing extra maternal heart components, this indeed happened in our experiments. The dimension of the subspace associated to the maternal ECG increased from 2 to 3 when instead of abdominal signals only, 3 abdominal signals were replaced by 3 thoracic signals. But whereas in the first case, much energy of the FECG was "lost" due to non orthogonality, in the second case this loss never occurred. See also [12], [13] for more details.

From the former section it is known already that the number of vector samples used to determine singular basis vectors must largely exceed the number of measurement signals. Otherwise, singular basis vectors are extremely sensitive to the actual noise signals. It seems contradictory, but even those time intervals during which neither the maternal nor the fetal heart seems to be active, are valuable to include in the set of measurement vector samples for the above reason. Some published methods used maternal QRS complexes only to determine the transfer vectors of the maternal heart, probably to reduce the required amount of calculations. For the reason given above this may result in large variations of singular basis vectors from one QRS complex to another. (The same holds of course for fetal QRS complex intervals). Further more, there is no guarantee that maternal ECG contributions at other instants (e.g. during atrial depolarization) remain within the subspace in which the QRS complex falls. Another disadvantage of such a procedure is that either human intervention and interpretation, or some automated recognition method is required. This renders such methods quite cumbersome since tests are to be done, and the actions to take become dependent on the actual data.

\section{Adaptive on-line method}

In the area of signal processing, two main approaches can be distinguished, respectively offline or on-line methods. In the off-line approach all data are collected first, and afterwards these data are processed. It has the advantage that different procedures can be compared using exactly the same data. In the online approach data are used as soon as they become available. And they are used exhaustively in the sense that these raw data need not be stored. Nevertheless a kind of summary of the past is kept in memory. The usual name for such a summary in systems theory is the state. Figure 4 illustrates schematically an on-line processing method.

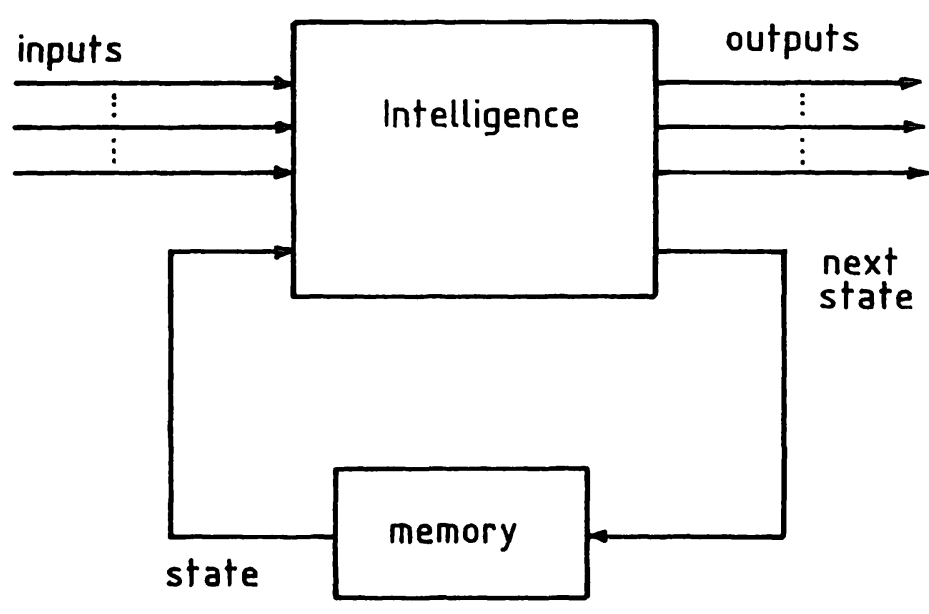

Figure 4. Block presentation of an on-line signal processing method. 
For a given off-line method, one could define the "equivalent" on-line method to be such that its output is equal to the output of the off-line method if only the raw data of the past were used. Not all off-line signal processing methods have such an economically feasible equivalent on-line method. The main constraints are the size of the required memory, and the pace set by the incoming data. This pace must be obeyed by the intelligence. The off-line method for FECG recording presented in [11], [12], [13] is based on the SVD as mentioned in the previous section. In [3], the authors have presented some mathematical aspects of an on-line strategy to carry out such an SVD. The inputs to the system are the measurement vector samples, the outputs are the weighted sums with the singular basis vectors as weight vectors. The state is represented as follows. It is a mathematical fact that the oriented energy in any direction for any set of vector samples can always be reconstructed, if only the singular basis vectors and the singular values are known. Therefore, these quantities constitute a relevant summary of the past, whatever the number of vector samples already taken. If $\mathbf{n}$ electrode signals are used, this requires in principle $n^{2}+n$ memory locations. The activity of the intelligence can be explained in geometrical terms as follows. Every time a measurement vector sample is presented at the input, the following actions are performed:

1) The measurement vector sample is projected onto each singular basis vector. The lengths of these projections are presented as outputs.

2) The singular basis vectors and singular values residing in the memory are updated, in order to contain the energy information of the last input vector sample.

It can be observed that in the procedure outlined above, the state represents the energy information from all vector samples from the past. There are some good reasons not to do so, for example if the transfer vectors are

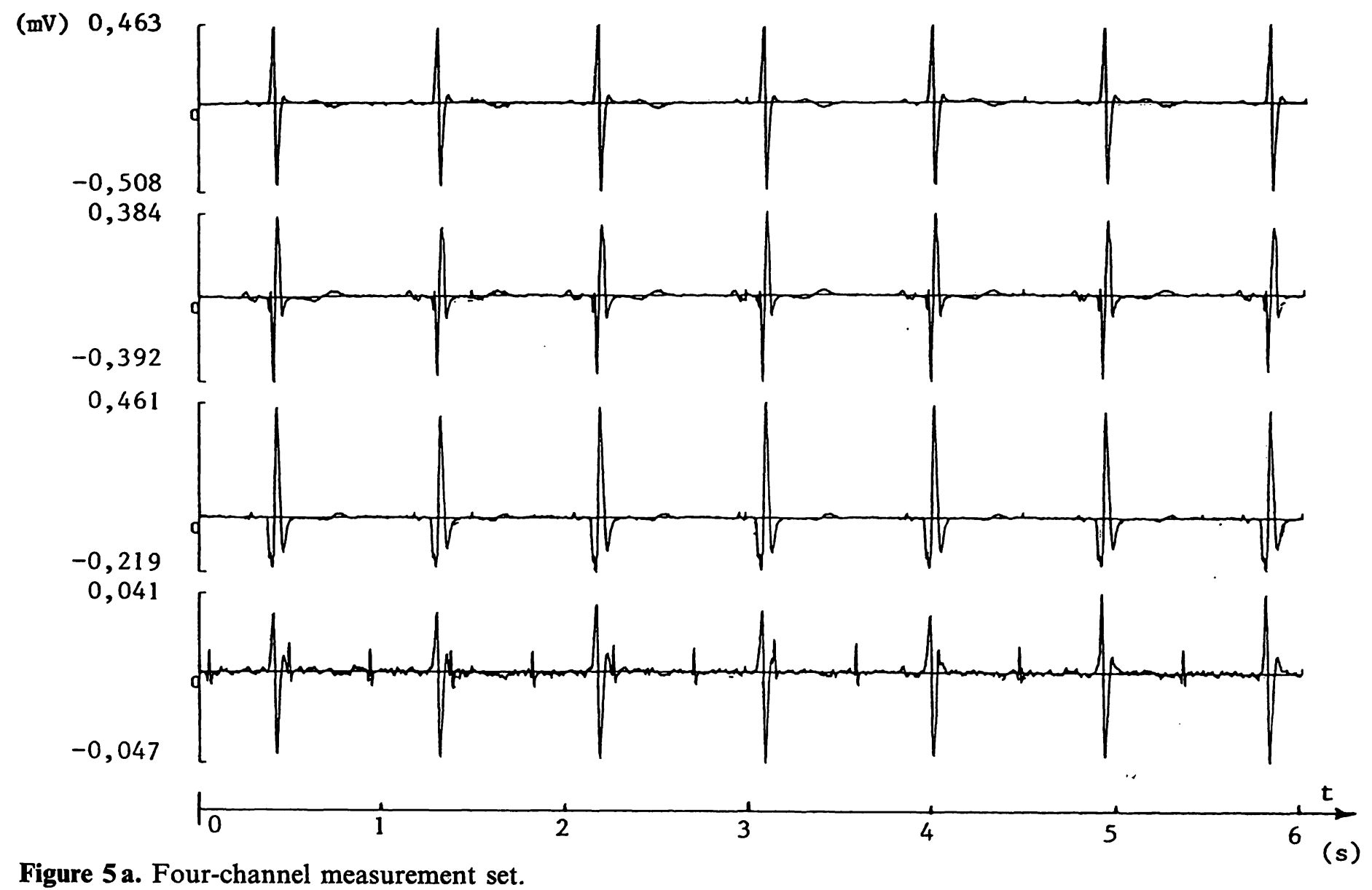

Figure 5 a. Four-channel measurement set. 
changing due to maternal breathing, or fetal movement. It would be better to use "more" from the near past, and "less" from the far past. An extremely simple way to accomplish this is by reducing, each time in step (2) above, the singular values slightly. In each time step, each singular value is multiplied by some factor $\alpha$ lying between 0 and 1 . It can be proved that this is equivalent to "exponential forgetting", i.e. the state is equal to the state that would have been obtained if vector samples from the past were exponentially weighted. The result is a so called adaptive method. If the transfer vectors change, the method tries to keep the output signal optimal. The smaller $\alpha$ is chosen, the faster the transfer vectors are allowed to change. However $\alpha$ should be large enough in order to limit the sensitivity to detailed short interval signal characteristics.

\section{Results and discussion}

Results presented here are obtained for the online adaptive method outlined in the previous sections by simulating it off-line. Except for the speed at which output signals become available, results must be identical however. For details about the measurement conditions and data collection the reader is referred to [11], [12].

A typical result is shown in figure 5. In figure 5 a the four input signals (i. e. electrode signals) are shown. The first three signals are from thoracic electrodes, the fourth signal is from an abdominal electrode. In figure $5 \mathrm{~b}$ the four resulting output signals are shown. Initially it can be seen that signals are still quite erroneous. But after about $1 \mathrm{~s}$ the fourth signal contains FECG virtually free of MECG. Indeed, after one MECG complex already enough oriented energy information has been aquired to find the fourth singular basis vector.

In general the results after about $1 \mathrm{~s}$ for this on-line method where essentially the same as the results for the off-line method, which are reported in [11], [12].

In figure 6 the result is shown of a test for the recovery after a sudden change. The method

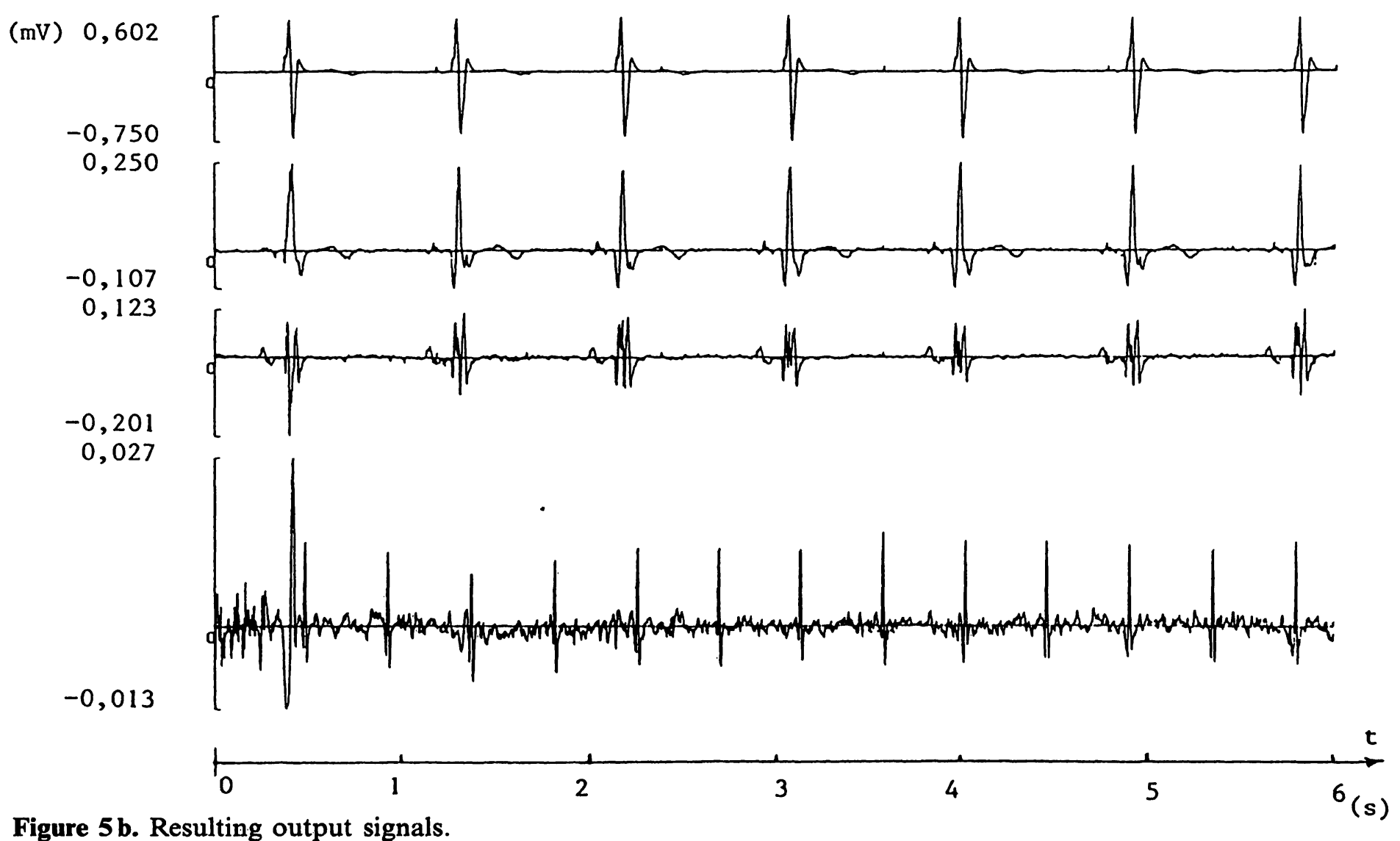

Figure 5 b. Resulting output signals. 
was started with 3 thoracic and 1 abdominal signal as inputs. After about $3 \mathrm{~s}$, the abdominal signal was replaced by another abdominal signal. Figure 6 a shows the abdominal signal, the arrow points to the instant of the change. Figure $6 \mathrm{~b}$ shows the fourth output signal. As can be seen, only 1 maternal QRS complex is required after the change to restore the separation. The fact that the resulting FECG becomes smaller is very normal, since the input abdominal signal obviously contained also less FECG. Figure 6 also shows that the problems of optimally reducing the MECG and optimally detecting the FECG are decoupled due to the use of abdominal and thoracic signals. This has been discussed in section 3 .

Figure 7 shows output signals of a test in which 3 thoracic and 3 abdominal signals were used as inputs. It shows that it is indeed dangerous to assume that fetal heart activity can be represented by only one signal. In other words, the fetal ECG as observed by cutaneous electrodes, can not always be represented by an equivalent current dipole with a fixed orientation.

\section{Conclusions}

A new method for the detection of the fetal ECG has been presented. This method is a further elaboration of an earlier presented offline technique [11], [12], [13].

As such it inherits the desirable properties of that technique. In principle it can be applied to measurement signals from any set of electrode location. There is however theoretical and ex-

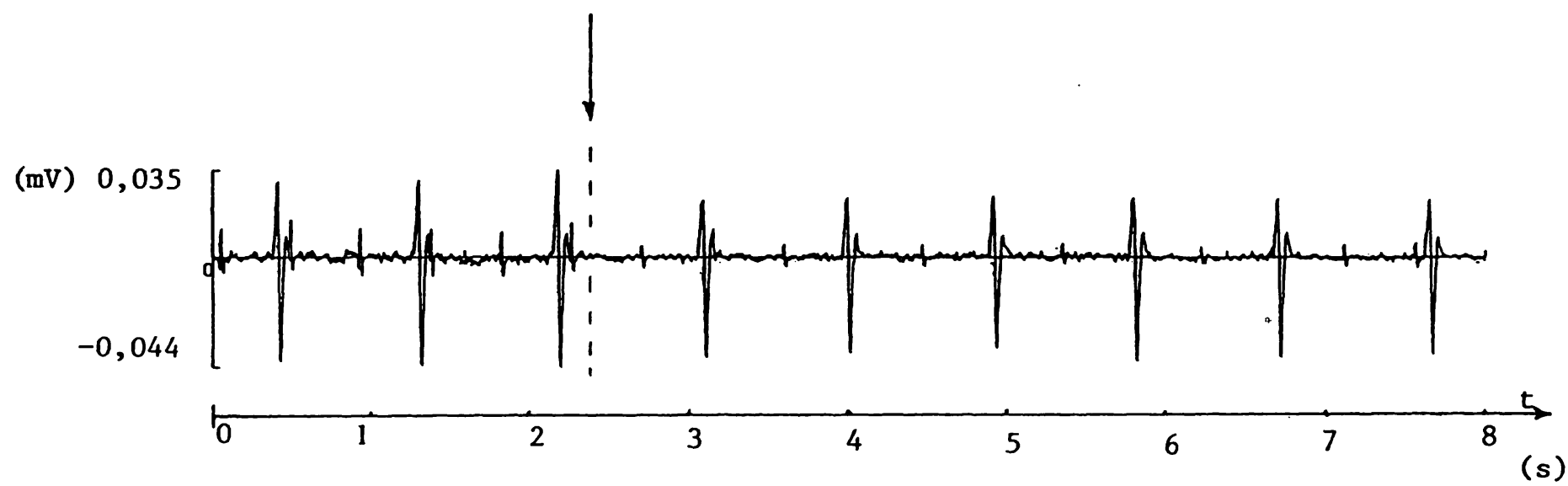

Figure 6 a. The abdominal signal: the arrow points where the sudden change to the other abdominal signal occured.

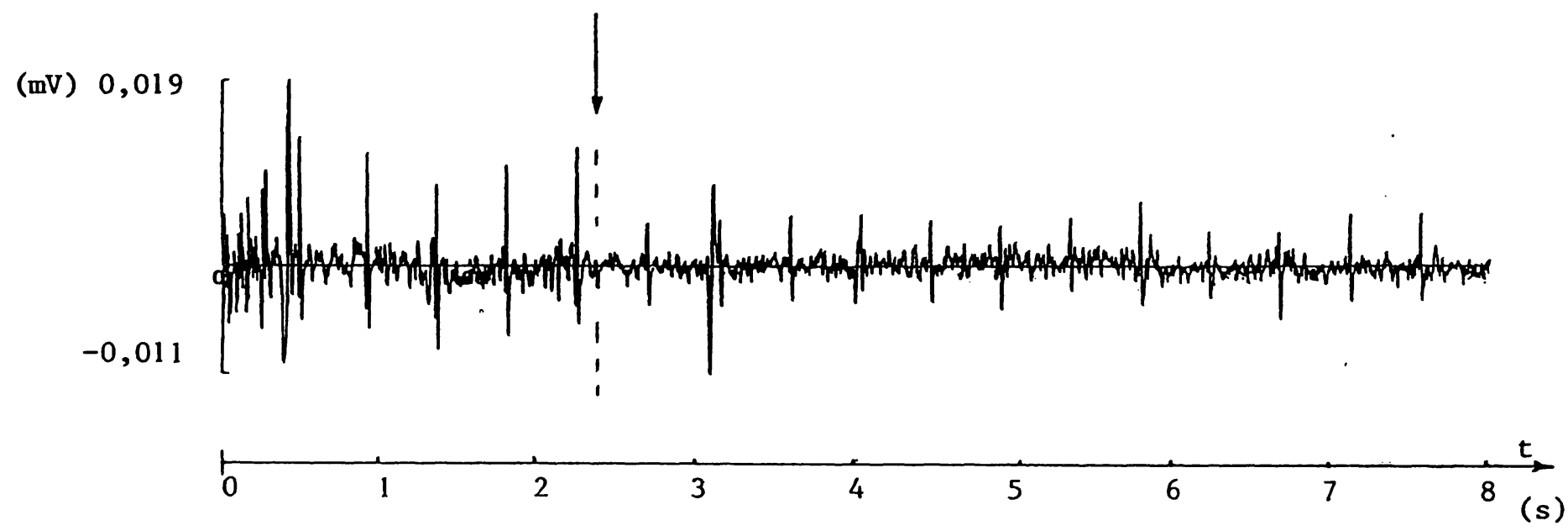

Figure $6 \mathrm{~b}$. The resulting fourth output signal. 
perimental evidence that the MECG can be suppressed to insignificant levels by using 3 thoracic signals as inputs. Other electrodes can then be put anywhere on the abdomen in order to obtain an optimal fetal ECG with respect to the noise. The idea is that it may be possible to find some standard positions on the abdomen, guaranteeing a minimal level of the detected FECG irrespective of gestational age or patients constitution.

Experimental evidence has been given that the cutaneous FECG may require more than one signal in order to be described completely, analogously to the adult ECG. The presented method allows for the detection of those additional signals.
The improvements obtained over the earlier off-line method, renders the on-line method attractive for clinical use by medical personnel. After $1 \mathrm{~s}$ a FECG, free of MECG, and with an optimal signal to noise ratio is obtained. After a sudden change of the signal character e. g. due to fetal movement, again only $1 \mathrm{~s}$ is required to recover from it. It is completely automatic, and requires no human intervention or interpretation.

At the time of writing, a module, based on a TMS 320 (Texas Instruments), and executing the presented on-line method for 8 input and 8 output signals at sample rates up to $500 \mathrm{~Hz}$, has been assembled and tested. This module will be used for a large scale evaluation in the hospital.

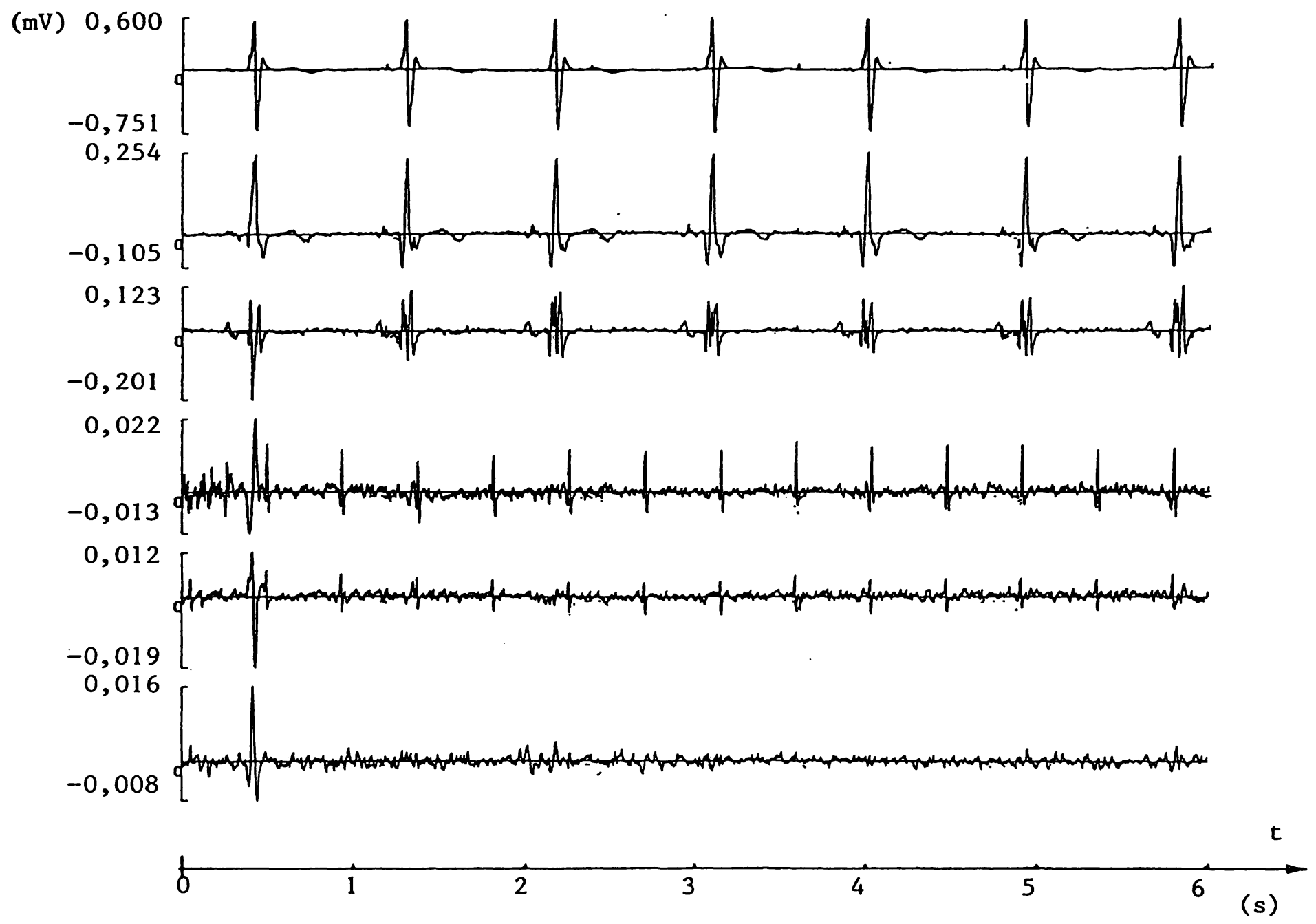

Figure 7. The output signals for a 6-channel measurement set showing two FECG-signals. 


\section{Summary}

The detection of a fetal electrocardiogram (FECG) is described to be a so-called inverse problem. A geometric interpretation of such a problem is presented. It can be solved by determining the weights to be used in one or more weighted sums of measurement signals. A novel method is presented to determine these weights. Theoretical and experimental evidence is given that this method has the following attractive properties. Application of the method results in one or more FECG signals, free of maternal ECG (MECG) which are at the same time maximal with respect to noise. It is shown that one FECG signal does not always suffice to describe the observed electrical heart activity in a set of cutaneous electrodes, equivalently to the adult ECG (see figure 7). It is explained and experimentally verified that the application of three thoracic signals is useful to guar- antee the suppression of the MECG. The positions of the abdominal electrodes then determine the obtained FECG to noise ratio, and will have no influence on the degree of MECG suppression (see figure 6). It is expected that a total of about eight measurement signals are sufficient in most cases. The method is simple to apply by medical personnel, and after a transient of about $1 \mathrm{~s}$ the signals with the mentioned properties are obtained (see figure 5). Further on, the weights are allowed to change in order to adapt automatically to sudden changes in the measurement signal properties (see figure 6). These may occur e.g. due to fetal movement. Application of the method requires no human interpretation or decision in order to obtain the claimed properties.

Keywords: Abdominal recording, adaptive on-line computation, fetal electrocardiogram, singular value decomposition, vector signals.

\section{Zusammenfassung}

Ableitung eines kompletten, fetalen EKG's aus multiplen, kutanen Aufzeichnungen mit einer adaptiven On-lineMethode

Die Ableitung eines fetalen Elektrokardiogramms (FEKG) kann als sogenanntes inverses Problem beschrieben werden. Wir geben eine geometrische Interpretation eines solchen Problems an. Es kann durch Bestimmung der Wertigkeiten in einer oder mehreren bewerteten Summen von gemessenen Signalen gelöst werden. Theoretisch und experimentell gibt es Hinweise, daß diese Methode folgende Vorzüge hat: Sie liefert ein oder mehrere FEKG-Signale, in denen das mütterliche EKG (MEKG) vollständig ausgeblendet ist und die ein maximales Nutz-/Störsignal-Verhältnis aufweisen. Es zeigt sich, daß bei Applikation mehrerer Hautelektroden ein einziges FEKG-Signal nicht immer genügt, um die elektrische Herzaktivität $\mathrm{zu}$ beschreiben, ähnlich wie beim
Erwachsenen-EKG (Abb. 7). Wir konnten experimentell nachweisen, daß bei Verwendung von 3 thorakalen Signalen das MEKG garantiert unterdrückt wird. Die Positionen der abdominalen Elektroden bestimmen dann das Verhältnis Nutz-/Störsignal und haben keinen Einfluß auf die Ausblendung des MEKG's (Abb. 6). Man kann erwarten, daß in den meisten Fällen ca. 8 Signale ausreichen. Die Methode ist durch das klinische Personal leicht zu handhaben; nach einer Aufnahmezeit von ca. 1 Sekunde sind die o.g. Signale meßbar (Abb. 5). Darüber hinaus kann die Wertigkeit der Signale verändert werden, um sich plötzlichen Abweichungen bei den Signalen automatisch anzupassen (Abb. 6), wie es z. B. bei fetalen Bewegungen notwendig wird. Die Anwendung der Methode erfordert somit keine Interpretation oder Entscheidung von Seiten des Personals.

Schlüsselwörter: Abdominale Ableitung, adaptive On-line-Verarbeitung, fetales Elektrokardiogramm, Vektorsignale, Zerlegung in Einzelwerte.

\section{Résumé}

Une méthode adaptative en temps réel pour l'extraction de l'électrocardiogramme fotal complet à partir d'enrégistrements par électrodes cutanées multiples

On décrit la détection de l'électrocardiogramme fotal (FECG) comme étant pour ainsi dire un problème inverse. On présente une interprétation géométrique d'un tel problème. Il peut être résolu en déterminant les intensités nécessaires pour une ou plusieurs sommes de signaux de mesure. On présente une nouvelle méthode de détermi- nation de ces intensités. Des preuves théoriques et expérimentales sont fournies que cette méthode a les propriétés séduisantes suivantes. L'application de la méthode a les propriétés séduisantes suivantes: L'application de la méthode fournit un ou plusieurs signaux de FECG, dégagé de l'ECG maternel (MECG) qui en même temps est maximal par rapport au bruit de fond. On montre qu'un signal du FECG ne suffit pas toujours pour décrire l'activité électrique cardiaque observée avec un ensem- 
ble d'électrodes cutanées, comme pour l'ECG adulte (voir figure 7). On explique et c'est vérifié expérimentalement que l'application de trois signaux thoraciques est utile pour garantir la suppression du MECG. Les emplacements des électrodes abdominales ensuite déterminent le rapport FECG obtenu sur bruit de fond, et n'influenceront nullement le degré de suppression du MECG (voir figure 6). On s'attend à ce qu'un total d'environ huit signaux de mesure suffisent dans la plupart des cas.
La méthode est simple à utiliser par le personnel médical, et au bout d'un délai d'environ $1 \mathrm{~s}$ on obtient le signal avec les propriétés mentionnées (voir figure 5). En outre, les intensités peuvent varier pour s'adapter automatiquement aux variations rapides des propriétés du signal de mesure (voir figure 6), ce qui peut survenir par exemple $\mathrm{du}$ fait des mouvements fœtaux. L'application de la méthode ne nécessite pas d'intervention humaine ni de décision pour l'obtention des propriétés affirmées.

Mots-clés: Décomposition en valeur singulière, électrocardiogramme fœtal, enregistrement abdominal, estimation adaptative en temps réel par ordinateur, signaux vectoriels.

\section{References}

[1] BEMMEL JH VAN: Detection of weak fetal electrocardiograms by autocorrelation and crosscorrelation of envelopes. IEEE Trans Biomed Eng 15 (1968) 17

[2] BergVeld P, WJH MeIJER: A new technique for the suppression of the MECG. IEEE Trans Biomed Eng 28 (1981) 348

[3] Callaerts D, J Vanderschoot, J Vandewalle: An on-line adaptive algorithm for signal processing using SVD. To be presented at EUSIPCO 86, The Hague, The Netherlands 1986

[4] DAMEN AA, J VAN DER KAM: The use of the singular value decomposition in electrocardiography. Med Biol Eng Comp 20 (1982) 473

[5] FERRARA ER, B WIDROW: Fetal electrocardiogram enhancement by time sequenced adaptive filtering. IEEE Trans Biomed Eng 29 (1982) 458

[6] Golub GH, CF vaN LoAN: Matrix Computations. North Oxford Acad Publ Co, Oxford 1983

[7] LONGINI RL, TA REICHERT, J MAN CHOYU, JS CROWLEY: Near-Orthogonal basis functions: a real time fetal ECG technique. IEEE Trans Biomed Eng 24 (1977) 39

[8] Oosterom A VAN, J Alsters: Removing the maternal component in the fetal ECG using singular value decomposition. In: RUTTKAY-NEDECKY I, P MACFARLANE (eds): Electrocardiology '83, Excerpta Medica, Amsterdam 1984

[9] PARLETT BN: The symmetric eigenvalue problem. Prentice Hall, Englewood Cliffs, N.J. 1980
[10] Plonsey R: Bioelectric Phenomena. Mc Graw-Hill, New York 1969

[11] Vanderschoot J, J Vandewalle, W Sansen, G VANTRAPPEN, J JANSSENS: An application of SVD to the extraction of weak bioelectrical signals. Revue HF XII (1983) 253

[12] VANDERSchoot J, G VANTRAPPEN, J JANSSENS, J VANDEWALLE, W SANSEN: A reliable method for fetal ECG extraction from abdominal recordings. In: Roger FH, JL Willems, R O'MOORE (eds): Medical Informatics Europe 84, Lecture Notes in Medical Informatics 24. Springer Verlag, Berlin 1984

[13] Vanderschoot J, J Vandewalle, J JansSens, W SANSEN, G VANTRAPPEN: Extraction of weak bioelectrical signals by means of singular value decomposition. In: Bensoussan A, JL Lions: Analysis and optimization of systems 1984, Lecture Notes in Control and Information Sciences 63, Springer Verlag, Berlin 1984

[14] WIDROW B, JR Glover, JM MCCOOL, J KanNITZ, ChS Williams, RM Hearn, JR Zeidler, E Dong, RC Goodlin: Adaptive noise cancelling: principles and applications. Proc IEEE 63 (1975) 1962

Dirk Callaerts

ESAT Laboratories

Katholieke Universiteit Leuven

Kardinaal Mercierlaan 94

B-3030 Heverlee, Belgium 